\title{
Thermography and microsystems-Some ways to intensify quantitative experimentation in heat transfer
}

\author{
by J.C. Batsale
}

TREFLE- ENSAM ; Esplanade des Arts et Métiers-33405 Talence cedex-France

\begin{abstract}
The microfabrication technique originally devoted to electronics have reached spectacular advances in more general domains such as chemical microreactors. The spatial temperature distribution of micro-electro-mechanical systems(MEMS) is among the most important information.

At small scales, the processing of the great amount of data given by temperature IR images is then offering challenging opportunities to quickly and simultaneously estimate a great amount of parameters (thermophysical properties, source terms distribution...).

Some examples will show that a convenient way is to consider a slow in-plane 2D transfer in a conductive plate at the surface of the system and to apply linear least square estimation principles.
\end{abstract}

\section{Introduction}

The aim of this lecture will consist in exploring the advantages of IR thermography observation in Micro-electro-mechanical systems(MEMS).

On the one hand, the microfabrication technique originally devoted to electronics have reach spectacular advances in general domains such as microreactors or microsensors (see [1], [2]). Such systems are very advantageous because the low fluid or solid mass requirements and small volumes induce low cost fabrication. It leads new ways to design and intensify the experiments such as screening for catalyst testing related to chemical engineering (see[3] ). Microdevices also have a lot of interesting characteristics in the field of heat transfer (high speed of the diffusive transfer, possibility of implementing localised heat sources, high surface/volume ratio). One of the main drawback is related to the instrumentation of such systems. Particularly, for temperature measurement, the implementation of small solid sensors is a difficult task.

On the other hand, the main advantage of IR thermography is to be non intrusive, offering possibilities of recording and processing a large amount of data related to heat transfer. The main challenge is then to set out processing methods of the surface temperature field of such systems through a heat transfer model in order to estimate the largest amount of parameters as possible (thermophysical properties, sources term distributions...).

Some of the principles of parameters estimation with IR thermography related to a heat transfer model will be first recalled. The aspects related to the noise effect reduction due to the linear processing of a large amount of data and the sensitivity analysis will be examined. Such principles will then be applied to simple well known situations at small scales. A first illustration will be to consider the case of in-depth 1D fast transfer classically applied to Non Destructive Evaluation of thin plates. Some important aspects related to the estimation in the case of in-plane diffusion and local diffusivity or conductivity parameter estimation will be further considered. Then, the perturbation of a in-plane macroscopic gradient due to convective transport in a 
micro-channel beside a conductive thin plate will be studied for the heat sources estimation, in the case of a chemical microreactor.

\section{Some considerations about the parameters estimation and IR thermography}

Technical considerations about IR cameras and IR microscopes are beyond the scope of this presentation. Only simple assumptions about focal plane array cameras will be considered. The studied phenomena will also be assumed to be always larger than the resolution limit of the thermographic device.

In the case of focal plane array camera, each detector of the array is considered to be unique (distinctive offset level and distinctive calibration coefficient), independent or not from the detectors positioned in the vicinity. Several apparatus present additive devices in order to make uniform the offset level and calibration coefficient of each detector of the array. The statistical description of the measurement noise associated to the signal proportional to the temperature can traduce such assumptions.

A signal associated to a pixel (proportional to a temperature) will be noted: $T$. The processing method will consist in estimating thermophysical properties of a considered sample. The estimation problem consists in comparing a theoretical response field $T(t, x, y)$ with experimental realisations noted: $\hat{T}(t, x, y)$.

The model in generally depending on a finite number of adjustable parameters noted: $\beta_{1}, \beta_{2}, \ldots \beta_{M}$ and of explicative variables such as time variable: $t$, or, space variables $x, y$. Each experimental realisation $\hat{T}\left(t_{i}, x_{j}, y_{k}\right)$ (component of vector: $\hat{\mathbf{T}}$ )is related to the theoretical model $T\left(t_{i}, x_{j}, y_{k}\right)$ (component of vector: $\mathbf{T}$ ) by the following:

$$
\hat{T}\left(t_{i}, x_{j}, y_{k}\right)=T\left(t_{i}, x_{j}, y_{k}\right)+e_{T\left(t_{i}, x_{j}, y_{k}\right)}
$$

$e_{T\left(t_{i}, x_{j}, y_{k}\right)}$ (component of vector: $\mathbf{e}_{\mathbf{T}}$ ) is assumed to be a random variable following a gaussian or normal distribution. With focal plane array cameras, the assumptions about the independence of these random variables depending on the time and space coordinates is questionable. Nevertheless, the assumption of independence of the pixels as a first approach allows to understand the processing methods implemented with simple expressions.

Generally the relation between parameters and temperature is non-linear, but the principle of all the optimisation methods is to describe the problems as iterative linear problems. Then, we will consider here only linear relations between parameters and temperatures such as the last step of an iterative optimisation method.

Then a summary of the simplified assumptions which will be used in the further applications is (see [4]):

1.Noise is additive: $\hat{\mathbf{T}}=\mathbf{T}+e_{\mathbf{T}}$

2. The relation between temperature and parameters to estimate is linear. $\mathbf{T}=\mathbf{X} \mathbf{B}$, with $\mathbf{X}$, sensitivity matrix independent from $\mathbf{B}$, parameter vector. 
3.The noise has a zero mean : $\mathbf{E}(\widehat{\mathbf{T}})=\mathbf{E}(\mathbf{T})$ or $\mathbf{E}\left(\mathbf{e}_{\mathbf{T}}\right)=\mathbf{0}$ (with $\mathbf{E}()$ : expected value operator)

4.Vector $\mathbf{B}$ is constant and unknown before the estimation.

5.Sensitivity matrix $\mathbf{X}$ is perfectly known

6.Error on $\mathbf{T}$ has a constant standard deviation $\sigma$

Such assumptions have been discussed by [4]. The optimal estimation of parameter vector $\mathbf{B}$ is then obtained by:

$$
\mathbf{B}=\left(\mathbf{X}^{\mathbf{t}} \mathbf{X}\right)^{-\mathbf{1}} \mathbf{X}^{\mathbf{t}} \mathbf{T}
$$

and the covariance matrix related to the estimation of $\mathbf{B}$ is:

$$
\operatorname{cov}(\mathbf{B})=\sigma^{2}\left(\mathbf{X}^{\mathbf{t}} \mathbf{X}\right)^{-1}
$$

This last expression allows to study the influence of the measurement noise about the parameter estimation. Expression (2) constitutes a linear transform of the data. In the case of the use of focal plane array, such linear transform can help to reduce the amount of information and at the same time the noise influence. For instance, if the observed signal is assumed to be stationary (the sensitivity matrix is then a unitary vector) the standard deviation of the unique parameter to be estimated is: $\sigma^{2} / N$ or $\sigma / \sqrt{N}$ ( $N$ is the number of realisations). The confidence interval of the estimation (proportional to the standard deviation) is then decreased when such a large amount of data is processed. The estimation is more difficult if the number of parameters $M$ is larger than one. The sensitivity matrix $\mathbf{X}$ must be such as $\left(\mathbf{X}^{\mathbf{t}} \mathbf{X}\right)$ is inversible or well conditioned. In the case of large amount of data (such IR thermography) the estimation of a large amount of parameters (smaller than the amount of data) is possible and often with a reduced noise influence, but the experimental conditions must be designed in order to dispose of a favourable sensitivity analysis. Particularly, the inversion of large linear systems is cumbersome and must be considered with suitable simplifications coming from the knowledge of the physical phenomena.

The following examples, will illustrate such principles.

\section{1D in-depth transfer}

Heat transfer can be considered as 1D in the case of study of thin plates and when the excitation is uniform on the faces of the plate. The pixels of the transient temperature images can then be assumed to be non correlated. The transient signal can then be processed pixel by pixel. Two families of excitation methods can then be considered : flash methods or periodic methods.

Periodic methods (see for example [5], [6]) consist in making a periodic thermal excitation at a surface of a sample.

Such methods have been used for the non destructive evaluation of defects in multilayered composite media. The excitation is generally realised with laser or lamps systems. The recording of the images is often synchronised with the transient excitation.

For example, a simple case such as semi-infinite medium can be considered. The heat transfer is assumed to be 1D (following $x$ ) and the thermal diffusivity: $a$ of the 
medium, does not depend on the temperature.

One example of temperature field inside the medium is then :

$$
T(x, t)=T_{m} \exp \left(-\sqrt{\frac{\omega}{2 a}} x\right) \cos \left(\omega t-\sqrt{\frac{\omega}{2 a}} x\right)
$$

The suitable linear transform $\mathbf{X}$ evocated in previous section can be here the Fourier transform following the time variable. Then, convenient estimation methods of the thermal diffusivity can be implemented by considering the amplitude variation of the signal, or the phase lag. It can be noticed that the phase lag is non depending on the absolute temperature level and can then be obtained without a calibration of the detectors.

The repetition of the same situation will reduce the noise influence by the superposition of the periods. Therefore, $N$ periods yields a decreasing of the noise influence following $\sqrt{N}$ Nevertheless, the repetition of experiments is not the lonely way to decrease the noise influence. For instance, when the characteristic frequency of the plate or the thermal diffusivity is unknown, even with thin samples, the flash method can be more advantageous than periodic methods. The power spectral density of heat source is then spreaded all over the frequencies. Other linear transforms than Fourier transform (other $\mathbf{X}$ sensitivity matrices) can be then considered such as autoregressive methods [7] or transform induced by Principal Component Analysis [8].

Besides, the pulsation $\omega$ or the characteristic time of the heating source is related to the characteristic penetration depth of the signal: $\sqrt{2 a / \omega}$. Therefore, the recording time step of the thermographic device at pixel scale must be adapted to such penetration depth. Unfortunately, at this moment, the recording time step is limited by the time necessary for the scanning or the recording of the whole image (tipically a few milliseconds). Such 1D methods are then non adapted to small scales (typically a glass or metallic plate lower than 0.1 millimeter depth).

\section{Slow in-plane diffusion}

1D in-depth heat transfer, previously considered, is valuable when the lateral sizes of the sample are far more larger than the thickness. Such assumption is not so evident with small scale micro-devices where complete 3D transfer in heterogeneous media must often be considered and where the in-depth diffusion can be toofast. One convenient solution is then to force slow in-plane heat transfer at the surface (instead of in-depth), by placing a conductive thin plate (more conductive than the substrate) in front and in contact with the micro-system. Such plate will act as a heat spreader. Generally a thin silicium wafer or a thin glass plate is used. At characteristic times larger than the in-depth diffusion time in the thin plate, the temperature field can be assumed to be $2 \mathrm{D}$. Only global lateral heat losses and source terms or perturbations coming from the substrate have then to be considered. The lateral heat losses are generally strong at larger scales (fin effect), but very small when a characteristic length $L$ (pixel size) of the conductive plate is such as: $L<<\sqrt{\lambda e / 2 h}$ (with $\lambda$ : thermal conductivity, $h$ convective losses coefficient, $e$ : thickness of the plate).

The analysis of the surface temperature needs to consider uniform emissivity and a uniform calibration of the pixels of the array (instead of in the previous 1D case), 


\section{http://dx.doi.org/10.21611/qirt.2006.a}

even if the signal can be only proportional to the absolute temperature.

2D heat transfer in thin plates has been studied with IR thermography in order to measure the global in-plane diffusivity of homogeneous samples (see [9]). The classical method consisted in analysing the overall image response to flash or periodic heat sources by spatial Fourier transform of the temperature images in order to obtain suitable expressions for the macroscopic diffusivityestimation. Such methods are very efficient when the sample is homogeneous and when the time scale is not too small or when the characteristic size of investigation is large enough. Unfortunately, the extension of spatial Fourier transform to heterogeneous samples is not easy and often it is difficult to take into account other phenomena as convective transport coupled with diffusion. The previous method has been extended at small scales. But local approaches, such as scanning with a laser hot spot (see[10]), are heavy to implement and allow to use only a small part of the possibilities of IR cameras (only a few pixels around the spot are sensitive and the scanning time of the global image is not adapted). The main part of the very large amount of available data is then useless.

Recently, local approaches have been implemented in our team in order to estimate the local field of diffusivity or conductivity of heterogeneous thin plates with IR cameras. The main idea is to simultaneously process the whole field of temperature and to try to make each pixel sensitive to local thermophysical parameters. Two examples are here briefly considered (a quasi steady method for estimation of local conductivities and a transient method with an initial spatially random field).

4.1 Quasi steady method for estimation of local conductivities of periodic in plane heterogenous samples (see [11])
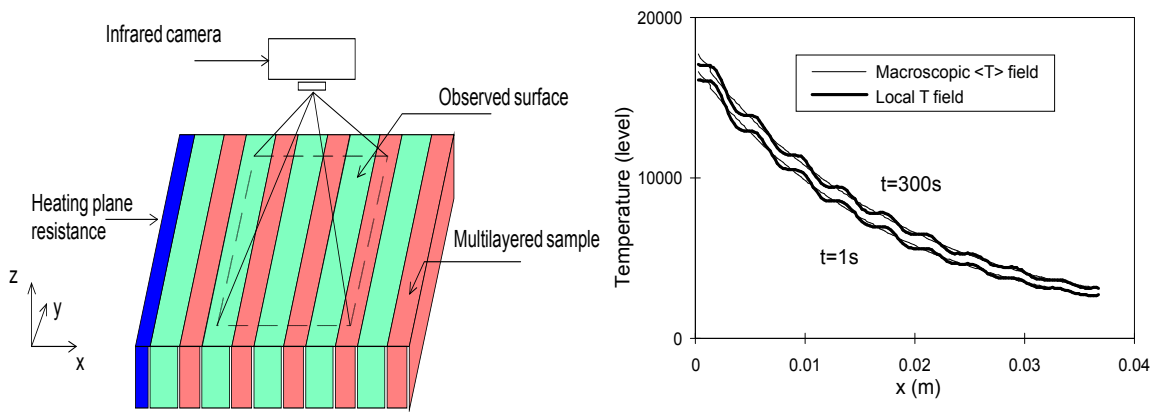

Fig 1: Scheme of the experimental device with one 1D heterogenous sample (the emissivity of the measured surface is uniform) and examples of temperature profiles.

Heterogenous media are here considered when the size of the heterogeneities is small compared to the global size of the sample to study. These media can be described with macroscopic parameters such as thermal conductivity or diffusivity, associated to a macroscopic model of the transfers. Homogenisation techniques as averaging methods (see [12]) can be used in order to theoretically justify such descriptions. Homogenisation methods can also be used as image processing technics associated with infrared images.

The basic idea of averaging homogenisation method is that a temperature field $T$ can 
be decomposed by the sum of a macroscopic field $\langle T\rangle$ obtained by moving average and a resultant local field $\widetilde{T}$. The relation between macroscopic and local field leads to a closure problem. It needs to consider a closure tensor $\boldsymbol{b}$ depending on the geometry of the heterogeneities and of the thermophysical properties of the media (see [12]), such as :

$\widetilde{T}=\boldsymbol{b} . \nabla\langle T\rangle$

It is here very important to remark that the sensitivity or the local component of the sensitivity matrix: $\mathbf{X}$ to a local conductivity change is then the macroscopic gradient $\nabla\langle T\rangle$. The optimal experiment will occur when the macroscopic gradients will be large. Such idea will be exploited in section 5 when small changes are caused by convection in micro-channels stick to a conductive plate.

4.2 Spatially random flash excitation for diffusivity mapping (see [13])

A spatially random flash excitation on the front face of a thin plate is here proposed as an alternative to the previous one. Only a biased but simple estimation method based on the application of Laplace operator is here presented. The main advantage is to allow the simultaneous process of a huge amount of data (varying versus space and time), sensitive all over the plate to the thermal conductivity mapping.

The method is devoted to the detection of cracks, which are considered as thin air layers of low thermal inertia, perpendicular to the observation surface.

A classical finite difference method is then used to solve the forward problem. A mesh of the 2D plate is composed of identical rectangular elements corresponding to a node of the system. At each node $(i, j)$ is corresponding a temperature $T_{i, j}^{t}$ at time $t$. By the same way a thermal diffusivity mapping $a_{i, j}$ is also defined at each node $(i, j)$. One approximation of the transient in-plane transfer is then given by the following vectorial expression:

$\mathbf{T}^{\mathbf{t}+\Delta t}-\mathbf{T}^{\mathbf{t}}=\mathbf{A} \cdot * \mathbf{T}$

$\mathbf{T}^{\mathbf{t}}$ is a temperature vector at time $t$, where the components are the pixels signal. $\Delta \mathbf{T}^{\mathbf{t}}$ is a vector obtained from the application of the Laplace operator to the previous vector $\mathbf{T}^{\mathbf{t}}$. The temperature mapping $\mathbf{T}^{\mathbf{t}+\Delta \mathbf{t}}$ is easily calculated at time $t+\Delta t$ from the knowledge of temperature mapping $\mathbf{T}^{\mathbf{t}}$ at time $t$. Among other, one "autoregressive " estimator of the vector A can then be implemented such as:

$\mathbf{A}=\left(\sum_{k=1}^{k_{\max }} \Delta \hat{\mathbf{T}}^{t_{k}} \cdot *\left(\hat{\mathbf{T}}^{t_{k+1}}-\hat{\mathbf{T}}^{t_{k}}\right)\right) \cdot\left(\sum_{k=1}^{k_{\text {max }}} \Delta \hat{\mathbf{T}}^{t_{k}} \cdot * \Delta \hat{\mathbf{T}}^{t_{k}}\right)$

Some experimental results are shown on figure 2.

The sensitivity is here locally the laplacian of temperature calculated on each pixel of the image. In order to be able to realize the estimation of the thermal diffusivity, it is absolutely necessary to create simultaneously non zero temperature laplacian and non-zero time derivative of the temperature field at each time step. The spatially random temperature field is then one of the best condition.

The principle of such method is advantageous, because it can be generalised to 


\section{http://dx.doi.org/10.21611/qirt.2006.a}

more complex models with more sophisticated estimation techniques (see application the Total Least Square estimation methods applications in [14] and [15]) and with sparse sensitivity matrices avoiding a global inversion of large linear systems.
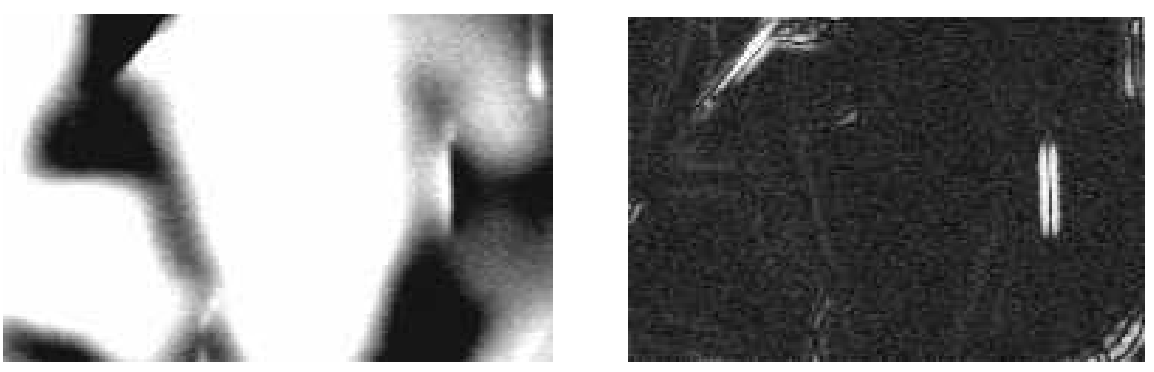

Fig 2 : Measured temperature field a few moment after the excitation and result of the estimation with expression (9).

\section{Processing of temperature fields in microreactors (see [16])}

This example is devoted to the analysis of temperature fields related to chemical microfluidic reactors. The previous ideas which consist in implementing a slow inplane transfer in a conductive plate is here used to estimate the convective transport effect due to a flow in a microchannel stick to the plate (see figure 3 ). The technical details of the experiment are described in [16]

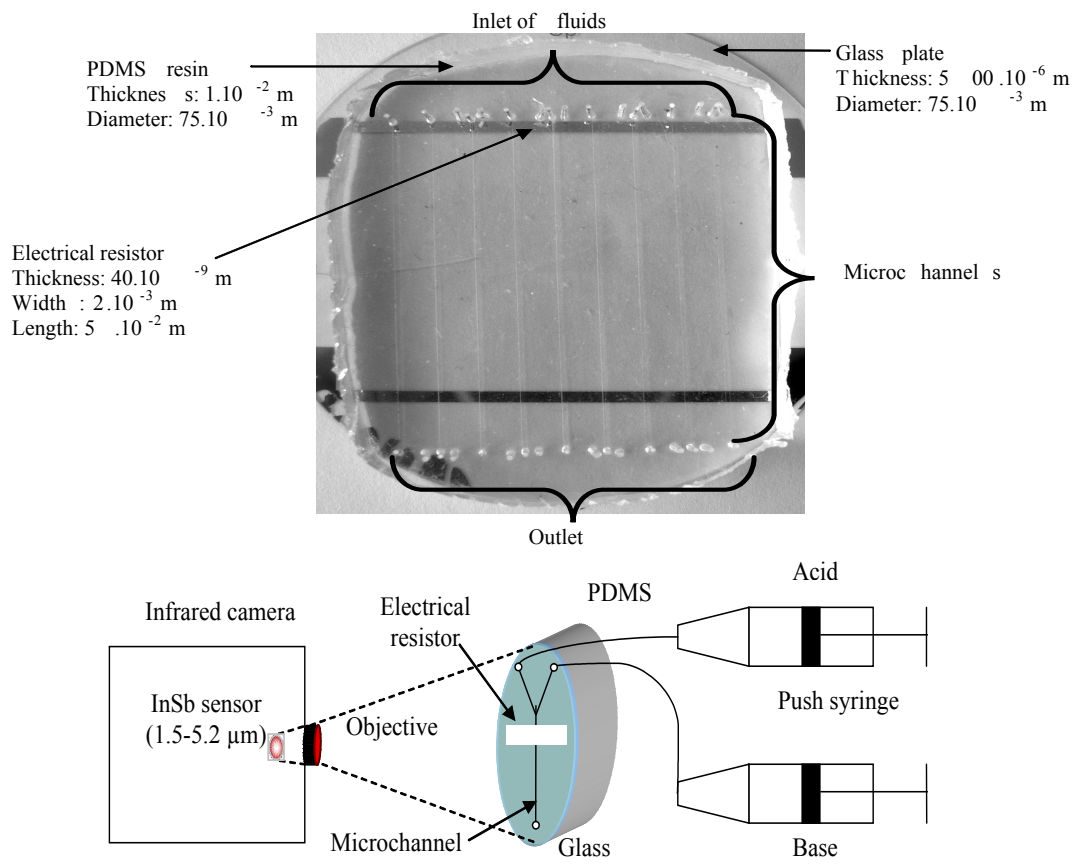

Fig. 3. Microfluidic chip, and principles of the microreactor experiment

The first step of the study will here consists in comparing and estimating the convective effect in the channel to the $2 \mathrm{D}$ diffusion inside the conductive front plate. 
Instead of the previous example in section 4, the local laplacian of the temperature field is compared to the local temperature gradient in order to estimate a Peclet Field. In order to realize a calibration experiment a thin heating resistor is deposited at the entrance of the channel on the conductive plate. A in-plane referencepermanent temperature field $T_{i, j}^{0}$ is obtained (see figure 4-a) with stationary pure water inside the channel and the heating on the left of the plate. Then, a perturbated temperature field $T_{i, j}$ is obtained with a water flow inside the channel (see Figure 4-b). The deformation of the isothermal lines is clearly shown by comparison between figure 4a and 4-b.
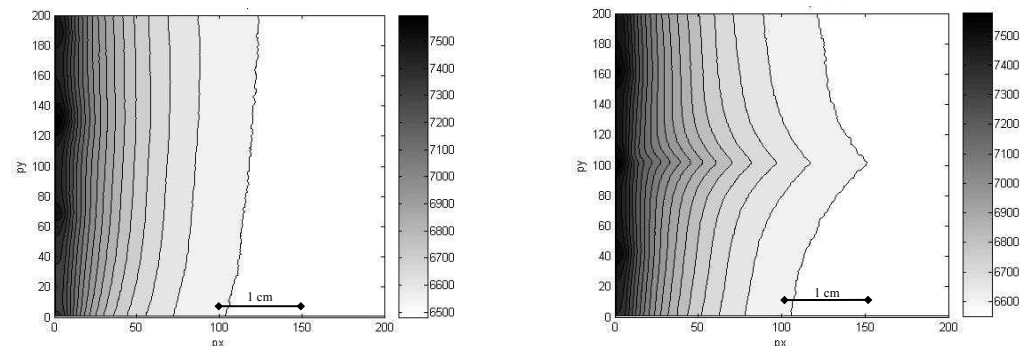

Fig 4:a-temperature field $T_{i, j}^{0}$ (without flow); b-temperature field $T_{i, j}$ (with micro flow)

In order to estimate the non dimensional velocity field compared to diffusion, a Peclet field $P e_{i, j}$ is locally defined such as:

$$
P e_{i, j}=\Delta\left(T_{i, j}-T_{i, j}^{0}\right) /\left(\partial\left(T_{i, j}\right) / \partial x\right)
$$

Once again the processing of the experimental temperature fields can be obtained with linear least square rules presented in section2.

It is very important to remark that such estimation (see figure 5) is giving a constant non zero velocity well estimated inside the channel location and with a poor noise only in the sharp temperature gradient zone.

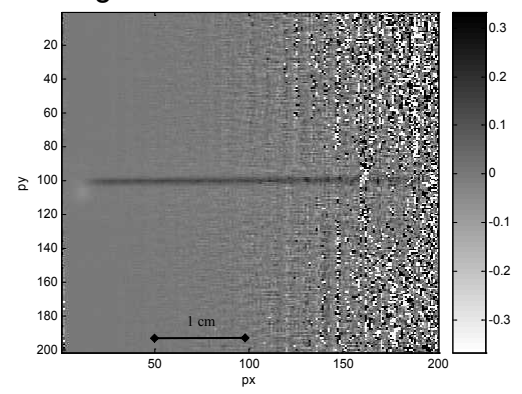

Fig. 5 Peclet field estimation $P e_{i, j}$ from figure 4

Such Peclet field can then be used in order to estimate the source term along the channel in the case of a specific microreactions in very near conditions of flow rate and fluids. Both liquids $(\mathrm{HCl}$ and $\mathrm{NaOH}$, concentration of $0.25 \mathrm{~mol} / \mathrm{l})$ are assumed to be with the same thermophysical properties as pure water. Then, the measurements are realised at the same flow rate as the previous experiment and the infrared camera allows the detection of the chemical heat flux created by the reaction. A 


\section{http://dx.doi.org/10.21611/qirt.2006.a}

temperature field measured in such conditions is shown on figure 6.

The distribution of source term estimation is then possible from the temperature field shown on figure 6 , with a further estimation and a overall calorimetric experiment explained in [16].

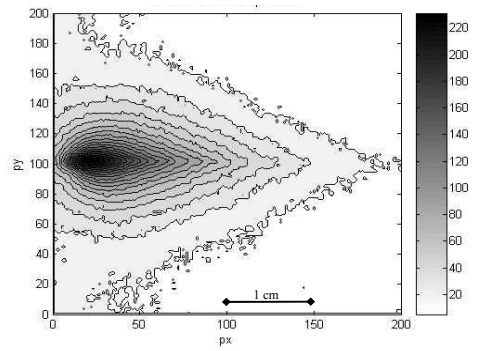

Fig. 6. Temperature field measurement $T^{c}{ }_{l, j}$ with an acid-base chemical reaction; concentration $0.25 \mathrm{~mol} / \mathrm{l}$ and flow rate $1000 \mu \mathrm{l} / \mathrm{h}$

\section{Conclusion-Perspectives}

The main advantage of such in-plane analysis of transfer at small scales is related to the very fast implementation of the experiments. Even if the first results obtained here are near from the steady-state, the stationary fields are generally obtained about a few seconds and authorizes a great number of trials (quite impossible at largerscales). The second aspect is related to the large possibilities to implement localised heat sources by external action (laser sources or heating resistors) or by observation of a tiny physical phenomena (chemical reaction, phase changes, electrothermal or thermomechanical coupling effects...). In the field of microfluidic experiments coupled with heat transfer a wide range of new applications will surely be developed (new intensive micro-scale heat exchangers, analysis of fluid-particles flows, flows at high temperature or hazardous conditions...).

A last illustration is shown on figure 7 related to flow of droplets of water transported by a non-miscible oil flow. Once again anoverall gradient is implemented in a conductive plate (here a silicon wafer) and the subtraction of the time averaged macroscopicfield allows to obtain with the same devices as in section 5 , the fluctuations of the temperature field. Such last field is clearly related to the instantaneous heat transfer between the droplet and the transporting oil flow.

Small sizes and great amount of information are the main idea of this presentation. Such last example illustrates how to intensify the experimentation productivity, by the possibilities to very quickly change the flow properties and the objects to be studied.
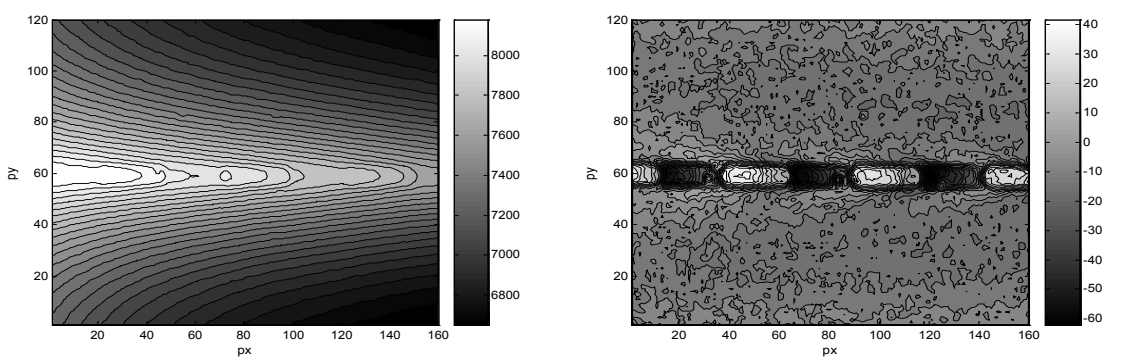

Fig 7: Water droplets in oil flow (channel width: 500um); a-Instantaneous temperature field; $b$ - Fluctuation field (obtained by subtraction of the previous field to the time averaged temperature field at 600 images per second). 


\section{Acknowledgements}

The main results presented here have been produced in a network of laboratories from Bordeaux and Toulouse universities (France). My colleagues (JL Battaglia, M. Bamford, M. Joanicot, D Mourand, C. Pradere, C Gourdon, O Fudym, J Toutain M Varenne) who participate to these works are greatly acknowledged.

\section{REFERENCES}

[1] K.F. Jensen, Microreaction engineering-is small better ?, Chemical Engineering Science, 2001, pp 293-303.

[2] K.P. Möllmann, N. Lutz and M. Vollmer, Thermography of microsystems, Inframation proceedings, ITC $104 \mathrm{~A}$, 07-27, 2004.

[3] S. Senkan, K. Krantz, S. Ozturk, V. Zengin, I. Onal, High-throughput Testing of Heterogeneous Catalyst Libraries Using Array Microreactors and Mass Spectrometry, Angewandte Chemie- International Edition, 38, 2794-2799, 1999

[4] Beck JV, Arnold KJ, Parameter estimation in engineering and science, John Wiley and Sons, NY, 1977.

[5] Wu D., Busse G., Lock-in thermography for nondestructive evaluation of materials Rev Gén de Thermique, Vol 37, 8, , 693-703 1998

[6] Wu D., Wu CY., Busse G., Investigation of resolution in lock in thermography- Theory and experiment, Eurotherm Quantitative Infrared Thermography, QIRT Stuttgart, 1996, pp 2-5.

[7] Mourand D., Batsale JC., Real time processing with low cost uncooled plane array IR camera-Application to flash non-destructive evaluation, QIRT Reims, 2000.

[8] Marinetti S, Grinzato E, Bison PG, Bozzi E, Chimenti M, Pieri G, O. Salvetti O.: Statistical analysis of IR thermographic sequences by PCA, Infrared Physics \& Technology, Vol 46, 1-2, 2004, 85-91

[9] Philippi I., Batsale JC, Maillet D., Degiovanni A., "Measurement of thermal diffusivity trough processing of infrared images" -Rev. Sci. Instrum. 66(1), 1995, pp182-192.

[10] Gruss C., Balageas D., "Theoretical and experimental applications of the flying spot camera“, Quantitative Infrared Thermography (QIRT 92), ED D. Balageas, G. Busse, GM Carlomagno, Ed Europ Therm. et Indus., Paris, 1992, pp19-24.

[11] Varenne M., Batsale J.C., "Infared images processing and volume averaging method in order to estimate 1D or 2D thermal conductivity fields". Image Analysis \& Stereologie, $\mathrm{N}^{\circ}$ 20 (2) supl. 1, 2001, 299-305.

[12] Quintard M. , Witaker S., One and two equations models for transient diffusion processes in two phases systems. Advances in Heat Transfer, 23, 369-464, 1993.

[13] Batsale JC, Fudym O, Battaglia JL, 2004, Autoregressive algorithms and spatially random flash excitation for $2 \mathrm{D}$ non-destructive evaluation with infrared cameras, QIRT journal, N1 pp1-20, 2004.

[14] Bamford M., Batsale JC., Rungoat D., Fudym O., Two dimensional velocity and diffusion mapping in the case of three dimensional transient diffusion: "Flash method and infrared image sequence analysis, QIRT 2006.

[15] Bamford M., Batsale JC., Mourand D., Bendada H., Detection of thermal bridges in insulating stratified media with thermography- A 2D transient direct model suitable to implement a suitable Total Least Square Estimation method, QIRT 2006.

[16] Pradère C., M. Joanicot, J.C. Batsale, J. Toutain, C. Gourdon, Processing of temperature field in chemical microreactors with infrared thermography, QIRT 2006. 